\title{
RENDIMIENTO EN PRUEBAS COGNITIVAS BREVES, DE ADULTOS MAYORES CON DEMENCIA EN ESTADIOS AVANZADOS, RESIDENTES DE UNA COMUNIDAD URBANA DE LIMA, PERÚ
}

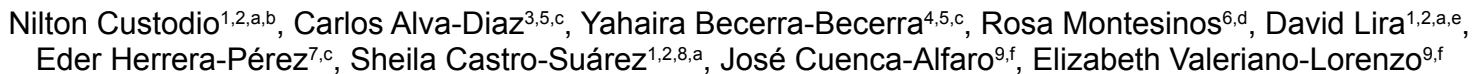

\begin{abstract}
RESUMEN
Objetivos. Evaluar el rendimiento de la prueba de dibujo de reloj en versión de Manos (PDR-M) y Mini Mental State Examination (MMSE) en su versión peruana, para detectar demencia de cualquier tipo en adultos mayores residentes de una comunidad urbana de Lima, Perú. Materiales y métodos. Análisis secundario de base de datos, de tipo observacional, analítico y transversal; con diseño de prueba diagnóstica, teniendo como prueba estándar a las evaluaciones clínica y neuropsicológica en conjunto. Se evaluó el rendimiento de las pruebas individualmente, así como de forma combinada. Resultados. El rendimiento de MMSE para la evaluación de pacientes con demencia de cualquier tipo presentó sensibilidad de $64,1 \%$, especificidad de $84,1 \%$, VPP de $24,4 \%$, VPN de $96,7 \%$, LR (+) de 4,03 y LR (-) de 0,43. La PDR-M presentó sensibilidad de $89,3 \%$, especificidad de $98,1 \%$, VPP de $79,3 \%$, VPN de $99,1 \%$, LR (+) de 47,79 y LR (-) de 0,11 . Cuando se aplicaron ambas pruebas, y al menos una de ellas fue positiva, presentaron sensibilidad de $98,1 \%$, especificidad de $84,1 \%$, VPP de $33,1 \%$, VPN de 99,8\%, LR (+) de 6,17 y LR (-) de 0,02. Al realizar el análisis, por separado, de demencia tipo alzhéimer y no alzhéimer, los valores de los parámetros no se diferencian sustancialmente de los obtenidos para demencias de cualquier tipo. Conclusiones. La combinación de MMSE y PDR-M demuestran un buen rendimiento para detectar demencia en estadios moderado y severo en población residente de una comunidad urbana de Lima.
\end{abstract}

Palabras clave: Demencia; Diagnóstico diferencial; Enfermedad de alzhéimer; Sensibilidad y Especificidad; Técnicas de Diagnóstico Neurológico (fuente: DeCS BIREME)

\section{PERFORMANCE OF COGNITIVE BRIEF TEST IN ELDERLY PATIENTS WITH DEMENTIA IN ADVANCED STAGE LIVING IN AN URBAN COMMUNITY OF LIMA, PERU}

\begin{abstract}
Objectives. Evaluate the performance of clock drawing test- Manos versión (PDR-M) and Mini Mental State Examination - Peruvian version (MMSE) to detect dementia in a sample based on urban community of Lima, Peru. Materials and methods. This study is a secondary analysis database, observational, analytical and cross-sectional, the gold standard was the clinical and the neuropsychological evaluations together. Performance testing individually and in combination were evaluated.. Data were obtained from prevalence study conducted in 2008 in Cercado de Lima. Results. MMSE performance for evaluation of patients with dementia of any kind showed sensitivity of $64,1 \%$, specificity of $84,1 \%$, PPV of $24.4 \%$, NPV of $96.7 \%$, PLR of 4,03 and NLR of 0,43 . PDR-M showed sensitivity of $89,3 \%$, specificity of $98,1 \%$, PPV of $79.3 \%$, NPV of $99.1 \%$, PLR of 47,79 and NLR of 0,11 . When both tests were applied, and at least one of them was positive, they showed sensitivity $98.1 \%$, specificity $84.1 \%$, PPV of $33.1 \%$, NPV of $99.8 \%$, PLR of 6,17 and NLR of 0,02 . When performing separate analysis of Alzheimer-type dementia and non- Alzheimer dementia, the values of the parameters do not differ substantially from those obtained for dementia of any kind. Conclussions. The combination of MMSE and PDR-M show good discriminative ability to detect moderate and severe dementia in population living in urban community in Lima.
\end{abstract}

Key words: Dementia; Differential diagnosis; Alzheimer's disease; Sensitivity and Specificity; Diagnostic Techniques, Neurological (source: MeSH NLM)

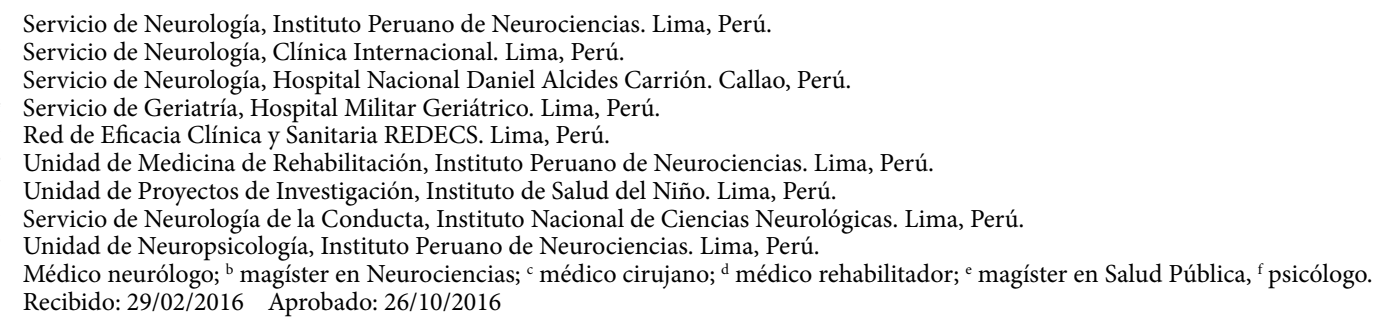

Citar como: Custodio N, Alva-Diaz C, Becerra-Becerra Y, Montesinos R, Lira D, Herrera-Pérez E, et al. Rendimiento en pruebas cognitivas breves, de adultos mayores con demencia en estadios avanzados, residentes de una comunidad urbana de Lima, Perú. Rev Peru Med Exp Salud Publica. 2016;33(4) 662-9. doi: 10.17843/rpmesp.2016.334.2549 


\section{INTRODUCCIÓN}

La demencia constituye un problema de salud pública, debido al envejecimiento poblacional, especialmente en América Latina ${ }^{(1,2)}$, donde la tasa global de demencia de ocho estudios latinoamericanos realizados en comunidad fue de $7,1 \%{ }^{(3)}$. En el Cercado de Lima, Perú (4) sobre 1532 individuos evaluados, se encontraron 105 casos de demencia, lo que corresponde a una prevalencia de $6,8 \%$, siendo la enfermedad de Alzhéimer (EA) el diagnóstico más frecuente $(56,2 \%)$.

El procedimiento diagnóstico de demencia requiere un protocolo especializado que incluye exámenes clínicos, de sangre e imágenes cerebrales y una amplia evaluación neuropsicológica, que deben ser realizados por especialistas en neuropsicología, escasos en Perú; por lo que existe una necesidad imperiosa de pruebas cognitivas breves (PCB) que puedan ser realizadas en los servicios primarios de atención, y que tengan adecuada sensibilidad y especificidad y mínima influencia del grado de instrucción. Las PCB además deben ser sensibles a los estadios iniciales de demencia, deben incluir como mínimo una evaluación de memoria episódica y funciones ejecutivas, dado que estos son los dominios cognitivos más afectados en las demencias más frecuentes como $E A^{(5)}$ y demencia vascular ${ }^{(6)}$.

Las PCB deben ser realizadas en diez minutos, o menos, y deben ser validadas en la región donde se van a emplear ${ }^{(7)}$. Las PCB con aceptables niveles de sensibilidad incluyen Memory Impairmente Screen $(\mathrm{MIS})^{(8)}$, prueba de dibujo del reloj (PDR) ${ }^{(9)}$, MiniCog Test ${ }^{(10)}$, test de los siete minutos ${ }^{(11)}$, Fototest ${ }^{(12,13)}$, Eurotest ${ }^{(14)}$ y el test de alteración de memoria $(\mathrm{T} @ \mathrm{M}){ }^{(15)}$; por otro lado, el Minimental State Examination (MMSE) es una de las pruebas cognitivas breves más utilizadas en el mundo para el tamizaje de pacientes con quejas cognitivas ${ }^{(16)}$. En Perú, solo se han validado PCB en individuos con alto nivel de instrucción, como la prueba de dibujo del reloj-versión de Manos (PDR-M) ${ }^{(17)}$, Addenbrooke's Cognitive examination $(\mathrm{ACE})^{(18)}$ y el test de alteración de memoria $(\mathrm{T} @ \mathrm{M})^{(19)}$; así también, en el caso del MMSE, diseñado originalmente en inglés, y posteriormente validado al español, se ha realizado la adaptación peruana del MMSE, en base a la versión rioplatense del MMSE de Butman et al. ${ }^{(20)}$. El punto de corte más eficaz para el ACE en la detección de casos con demencia en el estudio peruano fue de 86 sobre 100 , basado en la mayor sensibilidad y especificidad, y reforzado por el hecho de que, con este punto de corte, la asignación de demencia no depende del sexo, edad ni años de educación, pero el gran inconveniente es que requiere de personal altamente entrenado y se realiza en 40 minutos ${ }^{(18)}$.

Por otro lado, el T@M para discriminar EA de deterioro cognitivo leve amnésico, demostró una sensibilidad de $100 \%$ y especificidad de $98,9 \%$ (ABC: 1,000 ) con un punto de corte de $27,{ }^{(19)}$ y superiores a los obtenidos en una muestra de población española de Barcelona, con un promedio de 8 años de educación, donde un punto de corte de 28 tuvo una sensibilidad de $91 \%$ y especificidad de 78\% (ABC: 0,9300) ${ }^{(15)}$. El T@M puede realizarse en seis minutos, pero no debe ser usado en forma aislada para definir demencia, pues sus alteraciones reflejan deterioro cognitivo, sobre todo de memoria episódica, semántica y orientación, pero, no incluye evaluación de la funcionalidad, por lo que requiere pruebas funcionales breves que contengan evaluación de actividades de vida diaria. Así mismo, el T@M no evalúa otras áreas cognitivas, como memoria visual, lenguaje, habilidades visuoespaciales y funciones ejecutivas; por lo que puede perder la posibilidad de detectar otros tipos de deterioro cognitivo, como deterioro cognitivo leve no amnésico, EA atípicas (variante frontal y atrofia cortical posterior) y otras demencias del tipo no alzhéimer, como demencia frontotemporal, vascular, con cuerpos de Lewy y asociada a enfermedad de Parkinson. La PDR-M evalúa funciones ejecutivas (planificación, programación motora y ejecución) y algunos ítems de memoria, particularmente de tipo visual, y estableciendo un punto de corte de 7 se obtuvo una sensibilidad de $99,0 \%$ y una especificidad de $83,5 \%{ }^{(17)}$. EI MMSE es una PCB que evalúa brevemente orientación, recuerdo inmediato, atención y cálculo, recuerdo diferido, lenguaje y praxis constructiva.

El presente estudio tiene como objetivo evaluar el rendimiento de dos PCB: PDR-M (17) y MMSE en su versión peruana (16) para detectar demencia en una muestra basada en comunidad urbana del Cercado de Lima.

\section{MATERIALES Y MÉTODOS}

\section{DISEÑO Y POBLACIÓN DE ESTUDIO}

Se realizó un análisis secundario de base de datos, de tipo observacional, analítico y transversal; con diseño de prueba diagnóstica para conocer los indicadores de validez diagnóstica de las PCB en comparación con el estándar ideal de los diferentes tipos de demencia, la evaluación neuropsicológica. Los datos fueron obtenidos del estudio de prevalencia de demencia realizado el año 2008 por Custodio et al. en el cercado de Lima ${ }^{(4)}$. Un total de 43 entrevistadores, entre estudiantes de Medicina de la Universidad Nacional Mayor de San Marcos y Universidad Peruana Cayetano Heredia $(n=26)$, médicos generales recién graduados $(n=11)$ y residentes de la especialidad de Geriatría de la Universidad Nacional Mayor de San Marcos $(n=6)$, fueron entrenados apropiadamente por el investigador principal para realizar las pruebas de cribado. Los entrevistadores recibieron entrenamiento en la versión peruana del Mini-mental State Examination (MMSE) ${ }_{(16)}^{(19)}$ prueba de dibujo del reloj-versión de Manos (PDR-M) ${ }_{(21)}^{(16)}$ y Pfeffer Functional Activities Questionnaire (PFAQ) ${ }^{211}$, durante una semana antes de realizar las pruebas en el campo, después de lo cual fueron 
evaluados para establecer la confianza interevaluador, el cual fue establecido como superior a 0,90.

\section{POBLACIÓN Y MUESTRA}

Para el estudio primario, teniendo en cuenta una prevalencia esperada de $7 \%$ en adultos mayores de 65 años de edad, 2958 individuos deberían ser evaluados. Se realizó un muestreo bietápico por conglomerados. La unidad primaria de muestreo fueron las manzanas; la unidad secundaria de muestreo fueron las viviendas; las unidades de observación fueron las personas mayores de 65 años que cumplieron los criterios de inclusión de la fase I, las unidades informantes fueron las personas seleccionadas y los habitantes de la vivienda o los cuidadores habituales (en caso de ser requerido). Para determinar el número de unidades primarias de muestreo a seleccionar, se estableció que por cada manzana se seleccionara cuatro viviendas de manera aleatoria. Un supuesto que planteamos es que en cada vivienda habitan, en promedio, dos personas mayores de 65 años. Entonces, el número de unidades secundarias de muestreo a ser seleccionadas fueron 1479. Se seleccionaron 370 manzanas, distribuidas proporcionalmente al número de manzanas con que cuenta cada distrito de la jurisdicción de la dirección de Salud, Lima-Ciudad. Los casos fueron establecidos en individuos: 1. Con diagnóstico de demencia según

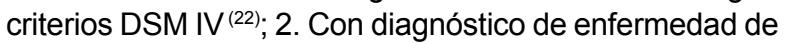
Alzhéimer según criterios de NINCDS-ADRDA (23); 3. Con diagnóstico de demencia vascular ${ }^{(24)}$; 4 . Con diagnóstico de demencia frontotemporal ${ }^{25)}$; y 5 . Con diagnóstico de demencia de enfermedad de Párkinson y demencia con cuerpos de Lewy ${ }^{(26)}$. Los controles fueron establecidos en individuos en los que: 1. Tras la evaluación clínica y neuropsicológica resultaron normales; 2. Se descartó pseudodemencia por presentar diagnóstico de depresión; y 3. Presentaron deterioro cognitivo explicado por otra causa: hipotiroidismo, deficiencia de vitamina B12, hepatopatía, nefropatía crónica, neuroinfecciones (infección asociada a VIH, sífilis), traumatismo cráneo encefálico severo, hematoma subdural, entre otras.

\section{INSTRUMENTOS DE RECOLECCIÓN DE DATOS}

El Mini Mental State Examination (MMSE) es una PCB que evalúa brevemente orientación (en tiempo y espacio), recuerdo inmediato (o registro de 3 palabras), atención y cálculo, recuerdo diferido (recuerdo retrasado o evocación), lenguaje (denominación, repetición, lectura, órdenes o comandos y escritura) y praxis constructiva. EI MMSE consta de 30 ítems, con valor de un punto para cada uno. El punto de corte para sospecha de demencia fue ajustado según años de educación: 27 para individuos con más de 7 años de educación, 23 para aquellos con 4 a 7 años de educación, 21 para aquellos con 1 a 3 años de educación, y 18 para los iletrados. Usamos la versión peruana, modificada de la de Buenos Aires ${ }^{(20)}$, realizada por Custodio y col ${ }^{(19)}$.
La prueba de dibujo del reloj - versión de Manos (PDR-M) evalúa las siguientes habilidades cognitivas: comprensión auditiva, planeamiento, memoria visual, reconstrucción de una imagen gráfica, habilidades visuoespaciales, programación motora y ejecución, conocimiento numérico y concentración. La PDR-M proporciona al evaluado, un círculo ya dibujado, con un área adecuada para graficar los elementos de la prueba. La interpretación se realiza de la siguiente manera: se traza una línea vertical que divide al círculo en dos mitades, teniendo como punto de partida el número 12 o el número 6; luego, se traza una línea perpendicular a la primera y, finalmente, se trazan dos diagonales, de tal manera que el círculo quede dividido en ocho áreas idénticas. Para la puntuación de la prueba no se toma en cuenta la posición de los números 3, 6, 9 y 12. Cada uno de los ocho números restantes debe estar incluido en cada una de las áreas. Por cada número ubicado en cada área se asigna un punto, de tal manera que el máximo puntaje posible es ocho puntos. El noveno punto se obtiene al valorar la direccionalidad de las agujas que representan el horario y el minutero dirigidos correctamente hacia los números 11 y 2 , respectivamente. Por último, el punto número diez se obtiene de la proporcionalidad correcta entre el horario y el minutero. El puntaje mínimo es cero y el máximo es 10 , un puntaje menor a 7 , indica compromiso cognitivo ${ }^{(16)}$.

El Pfeffer Functional Activities Questionnaire (PFAQ) incluye once preguntas acerca de las actividades de la vida diaria, con rangos de puntaje de 0 a 3 según la severidad de la discapacidad en cada actividad. El puntaje máximo es 33, y un uno mayor de 6 indica compromiso funcional ${ }^{(21)}$.

Los criterios de inclusión fueron establecidos en individuos: 1. De sexo masculino y femenino con edades superiores a 60 años de edad; y 2. Que aceptaran participar en el estudio y firmaran el consentimiento informado. Los criterios de exclusión fueron establecidos en individuos con: 1. Dificultad para realizar las pruebas cognitivas, debido a problemas auditivos, visuales $u$ otros problemas físicos que pudieran interferir con su rendimiento; 2. Lengua diferente al español; y 3. Antecedentes de adicción o abuso de sustancias.

\section{PROCEDIMIENTO}

Los individuos evaluados fueron sometidos a las siguientes valoraciones sucesivas (cribado, diagnóstico y tipo de demencia) en cada fase.

Fase de cribado de demencia: los individuos fueron sometidos a evaluación clínica integral y a PCB que incluyeron MMSE, PDR-M y PFAQ.

Fase de diagnóstico de demencia: los individuos que tuvieron un rendimiento por debajo de los puntajes 
establecidos para este protocolo de investigación fueron sometidos a una segunda evaluación, en la cual se efectuó un segundo MMSE y PDR-M por un evaluador distinto al de la fase de cribado. Los individuos que confirmaron "deterioro cognitivo" en la segunda evaluación, fueron sometidos a exámenes sanguíneos, tomografía cerebral y/o resonancia magnética cerebral, descarte de depresión, mediante aplicación del inventario de depresión de Beck-II (BDI-II); con lo cual se realizó el descarte de pseudodemencia y, por otro lado, se realizó la confirmación del diagnóstico de demencia y fueron derivados para la evaluación neuropsicológica, la cual es una evaluación detallada de las funciones cognitivas, mediante una batería neuropsicológica adaptada a nuestra población. La evaluación neuropsicológica para esta investigación incluyó los siguientes test: Rey Auditory Verbal Learning Test ${ }^{(27)}$, Logical Memory Subtest del Weschler Memory Scale revisado ${ }^{(28)}$, Trail Making Test $A$ y $B^{(29)}$, figura compleja de Rey ${ }^{(27)}$, test de denominación de Boston ${ }^{(30)}$, Wisconsin Card Sorting Test (WCST) ${ }^{\left({ }^{31}\right)}$, y Letter- Number (subtest del Weschler Adult Intelligent Scale III) ${ }^{(28)}$.

Fase de tipo de demencia: en esta fase, con los resultados de los exámenes de sangre, de imágenes cerebrales y el informe neuropsicológico, los investigadores, neurólogos y neuropsicólogos, expertos en demencias, realizaron el diagnóstico por tipo de demencia, y estuvieron ciegos a los resultados de las PCB. En caso de dificultad, hubo una evaluación y discusión conjunta.

Estadio de demencia: para evaluar la severidad de la demencia, empleamos la escala denominada Clinical Dementia Rating (CDR), que tiene mayor sensibilidad en la detección de cambios cognitivos que las pruebas cognitivas con normas interindividuales, pero su aplicación requiere más tiempo y suficiente entrenamiento para la práctica clínica habitual. La escala CDR, habitualmente, es utilizada en casos de diagnósticos ya establecidos de demencia, y es útil para determinar el nivel de severidad; siendo ampliamente empleada en estudios epidemiológicos, series de casos y ensayos clínicos, utilizando para este estudio la versión peruana de $\mathrm{CDR}^{(32)}$.

Con la finalidad de realizar los cálculos de rendimiento de las PCB se establecieron los siguientes grupos diagnósticos:

- Demencia de cualquier tipo: incluye a personas con diagnóstico de EA probable y posible, demencia vascular (DV), EA con enfermedad cerebrovascular (EA+ECV), demencia por enfermedad de Parkinson (DEP), demencia con cuerpos de Lewy (DCLewy) y demencia frontotemporal (DFT).
- Demencia tipo alzhéimer: incluye a personas con diagnóstico de EA probable y posible.

- Demencia no alzhéimer: incluye a personas con diagnóstico de DV, EA+ECV, DEP, DCLewy y DFT.

\section{ANALISIS ESTADÍSTICO}

Se realizaron los cálculos para medidas de frecuencia, de tendencia central y dispersión. Para conocer el rendimiento de las $\mathrm{PCB}$ se realizaron los cálculos comparando cada una de las PCB así como las diferentes combinaciones de ellas frente al gold estándar que estuvo dado por las evaluaciones clínica y neuropsicológica en conjunto, teniéndose en cuenta la prevalencia de demencia $(7,42 \%)$ en una población urbana de Lima. En cuanto a las combinaciones de PCB, se consideró como test positivo cuando, al menos, una de las PCB fue positiva. Es así que se determinaron los siguientes indicadores para cada comparación: sensibilidad (S), especifidad (E), valor predictivo positivo (VPP), valor predictivo negativo (VPN), proporción de falsos positivos, proporción de falsos negativos, cociente de probabilidad positivo (CPP) o Likehood Ratio positivo (LR+) y cociente de probabilidad negativo (CPN) o Likehood Ratio negativo (LR-), exactitud, odds ratio diagnostico e índice $\mathrm{J}$ de Yauden. Para llevar a cabo el análisis de los datos se utilizó el paquete estadístico STATA versión 14 mediante la función diagti. Los contrastes se realizarán con un nivel de confianza del $95 \%$ y un nivel de significancia del $5 \%$. Se realizaron cálculos de los valores puntuales, así como sus intervalos de confianza al $95 \%$.

\section{CONSIDERACIONES ÉTICAS}

El estudio fue aprobado por el comité de ética y técnicoinstitucionales de la Clínica Internacional y de la Universidad de San Martín de Porres. La participación de la población fue voluntaria, y todos ellos fueron visitados previamente para obtener un consentimiento informado directamente del individuo evaluado o de un miembro de la familia o del cuidador habitual.

\section{RESULTADOS}

La población estuvo constituida por 210 adultos mayores (103 pacientes con diagnóstico de demencia y 107 sanos) de la segunda fase del estudio de prevalencia de demencia en el Cercado de Lima, con una edad promedio de 81,6 (DE: 6,98), constituyendo el mayor porcentaje el grupo etario de 85 a 96 años con $38,6 \%$. Respecto al sexo, se observa un porcentaje mayor del femenino $(70,5 \%)$ con diferencia significativa $(p<0,01)$ con respecto al sexo masculino. En cuanto a los años de educación, los individuos se distribuyeron uniformemente en los diferentes niveles, siendo $24,8 \%$ de ellos iletrados (Tabla 1). 
Tabla 1. Distribución según edad, años de educación y sexo de pacientes con diagnóstico de demencia según evaluación neuropsicológica en Cercado de Lima

\begin{tabular}{|c|c|c|c|}
\hline & $\mathrm{n}(\%)$ & Femenino (\%) & Masculino (\%) \\
\hline \multicolumn{4}{|c|}{ Grupo de edad } \\
\hline 65 a 69 & $15(7,1)$ & $10(66,7)$ & $5(33,3)$ \\
\hline 70 a 74 & $30(14,3)$ & $19(63,3)$ & $11(36,7)$ \\
\hline 75 a 79 & $31(14,8)$ & $23(74,2)$ & $8(25,8)$ \\
\hline 80 a 84 & $53(25,2)$ & $39(73,6)$ & $14(26,4)$ \\
\hline 85 a 96 & $81(38,6)$ & $57(70,4)$ & $24(29,6)$ \\
\hline \multicolumn{4}{|c|}{ Años de educación } \\
\hline lletrados & $52(24,8)$ & $37(71,2)$ & $15(28,9)$ \\
\hline 1 a 3 & $58(27,6)$ & $44(75,9)$ & $14(24,1)$ \\
\hline 4 a 7 & $48(22,9)$ & $30(62,5)$ & $18(37,5)$ \\
\hline 8 a más & $52(24,8)$ & $37(71,2)$ & $15(28,9)$ \\
\hline Total & $210(100)$ & $148(70,5)$ & $62(29,5)$ \\
\hline
\end{tabular}

En cuanto a la distribución de los casos de demencia encontrados en esta población, los más altos porcentajes corresponden a la EA probable con $49,5 \%$, seguida por la $E A+E C V$ con un $15,5 \%$, siguiendo luego DV $(8,7 \%)$, EA posible $(6,8 \%)$, DEP $(2,9 \%)$, DCLewy $(1,9 \%)$ y DFT $(1,9 \%)$; así mismo se observó un alto porcentaje del diagnóstico de demencia de tipo no determinado. Según la escala CDR, $41(39,8 \%)$ pacientes fueron clasificados como demencia leve (CDR 1), 36 (34,9\%) como moderada (CDR 2), y 26 (25,3\%) como severa (CDR 3).

\section{ANALISIS DEL RENDIMIENTO DE LAS PRUEBAS COGNITIVAS BREVES}

Respecto al rendimiento de las PCB para la evaluación de pacientes con demencia de cualquier tipo, el MMSE presentó una sensibilidad de $64,1 \%$, una especificidad de $84,1 \%$, un VPP de $24,4 \%$, un VPN de $96,7 \%$, un CPP o LR (+) de 4,03 y un CPN o LR (-) de 0,43. La PDR-M presentó una sensibilidad de $89,3 \%$, una especificidad de $98,1 \%$, un VPP de $79,3 \%$, un VPN de $99.1 \%$, un CPP o LR (+) de 47,79, un CPN o LR (-) de 0,11. Cuando se aplicaron ambas pruebas (MMSE y PDR-M), y al menos una de ellas fue positiva, presentaron una sensibilidad de $98,1 \%$, una especificidad de $84,1 \%$, un VPP de $33,1 \%$, un VPN de $99,8 \%$, un CPP o LR (+) de 6,17 y un CPN o LR (-) de 0,02 (Tabla 2).

Evaluando el rendimiento de las PCB para la evaluación de pacientes con demencia tipo alzhéimer el MMSE presentó una sensibilidad de $60 \%$, una especificidad de $84,1 \%$, un VPP de $23,2 \%$, un VPN de $96,3 \%$, un CPP o LR (+) de 3,8 y un CPN o LR (-) de 0,5. La PDR-M presentó una sensibilidad de $90 \%$, una especificidad de $98,1 \%$, un VPP de $79,4 \%$, un VPN de 99,2\%, un CPP o LR (+) de 48,2, un CPN o LR (-) de 0,1. Cuando se aplican ambas pruebas (MMSE y PDR-M), y al menos una de ellas fue positiva, presentaron una sensibilidad de $98,3 \%$, una especificidad de $84,1 \%$, un VPP de $33,2 \%$, un VPN de $99,8 \%$, un CPP o LR (+) de 6,19, un CPN o LR (-) de 0,02 (Tabla 3).

En el rendimiento de las PCB para la evaluación de pacientes con demencia tipo no alzhéimer el MMSE presentó una sensibilidad de $80 \%$, una especificidad de $84,1 \%$, un VPP de $28,8 \%$, un VPN de $98,1 \%$, un CPP o LR (+) de 5 y un CPN o LR (-) de 0,2. La PDR-M presentó una sensibilidad de $86,7 \%$, una especificidad de $98,1 \%$, un VPP de $78,8 \%$, un VPN de $98,9 \%$, un CPP o LR (+) de 46,4, un CPN o LR (-) de 0,1. Cuando se aplican ambas pruebas (MMSE y PDR-M), y al menos una de ellas fue positiva, presentaron una sensibilidad de $93,3 \%$, una especificidad de $84,1 \%$, un VPP de $32.0 \%$, un VPN de $99,4 \%$, un CPP o LR (+) de 5,87, un CPN o LR (-) de 0,08 (Tabla 4).

Tabla 2. Rendimiento de pruebas cognitivas breves aplicadas en pacientes con demencia de cualquier tipo en Cercado de Lima $(n=210)$

\begin{tabular}{|c|c|c|c|c|c|c|}
\hline \multirow{2}{*}{ Indicador } & \multicolumn{2}{|c|}{ MMSE } & \multicolumn{2}{|c|}{ PDR-M } & \multicolumn{2}{|c|}{ MMSE Y PDR-M } \\
\hline & $\%$ & IC $95 \%$ & $\%$ & IC $95 \%$ & $\%$ & IC $95 \%$ \\
\hline Sensibilidad & $64,1 \%$ & $54,5-72,7$ & 89,3 & $81,9-93,9$ & 98,1 & $93,2-99,5$ \\
\hline Especificidad & 84,1 & $76-89,8$ & 98,1 & $93,4-99,5$ & 84,1 & $76-89,8$ \\
\hline Valor predictivo positivo & 24,4 & $17,0-33,8$ & 79,3 & $49,2-93,8$ & 33,1 & $24,2-43,4$ \\
\hline Valor predictivo negativo & 96,7 & $95,7-97,5$ & 99,1 & $98,5-99,5$ & 99,8 & $99,3-100,0$ \\
\hline Área ROC (exactitud) & 0,74 & $0,68-0,80$ & 0,94 & $0,90-0,97$ & 0,91 & $0,87-0,95$ \\
\hline Odds ratio diagnóstica & 9,4 & $4,92-18,12$ & 439,09 & $94,85-2032,76$ & 267,35 & $60,1-1189,22$ \\
\hline $\operatorname{LR}(+)$ & 4,0 & $2,55-6,38$ & 47,79 & $12,09-188,91$ & 6,17 & $3,99-9,55$ \\
\hline $\operatorname{LR}(-)$ & 0,4 & $0,32-0,56$ & 0,11 & $0,06-0,19$ & 0,02 & $0,01-0,09$ \\
\hline
\end{tabular}


Tabla 3. Rendimiento de pruebas cognitivas breves aplicadas en pacientes con demencia de tipo alzhéimer en Cercado de Lima $(n=210)$

\begin{tabular}{|c|c|c|c|c|c|c|}
\hline \multirow{2}{*}{ Indicador } & \multicolumn{2}{|c|}{ MMSE } & \multicolumn{2}{|c|}{ PDR-M } & \multicolumn{2}{|c|}{ MMSE Y PDR-M } \\
\hline & $\%$ & IC $95 \%$ & $\%$ & IC $95 \%$ & $\%$ & IC $95 \%$ \\
\hline Sensibilidad & 60,0 & $47,4-71,4$ & 90,0 & $79,9-95,3$ & 98,30 & $91,1-99,7$ \\
\hline Especificidad & 84,1 & $76-89,8$ & 98,1 & $93,4-99,5$ & 84,10 & $76-89,8$ \\
\hline Valor predictivo positivo & 23,2 & $15,7-32,9$ & 79,4 & $49,4-93,9$ & 33,2 & $24,3-43,4$ \\
\hline Valor predictivo negativo & 96,3 & $95,0-97,3$ & 99,2 & $98,3-99,6$ & 99,8 & $98,9-100,0$ \\
\hline Área ROC (exactitud) & 0,72 & $0,65-0,79$ & 0,94 & $0,90-0,98$ & 0,91 & $0,87-0,95$ \\
\hline Odds ratio diagnóstica & 7,9 & $3,82-16,51$ & 472,5 & $92,24-2420,39$ & 312,35 & $40,48-2410,3$ \\
\hline $\operatorname{LR}(+)$ & 3,8 & $2,33-6,12$ & 48,2 & $12,17-190,53$ & 6,19 & $4-9,58$ \\
\hline $\operatorname{LR}(-)$ & 0,5 & $0,34-0,66$ & 0,1 & $0,05-0,22$ & 0,02 & $0-0,14$ \\
\hline
\end{tabular}

MMSE: Mini Mental State Examination. PDR-M: prueba de dibujo del reloj - versión de Manos. LR(+): Likehood ratio positivo. LR(-):Likehood ratio negativo

\section{DISCUSIÓN}

La muestra estudiada es de predominio del sexo femenino, lo cual no afecta las conclusiones del estudio; pues esta representa la misma proporción obtenida en los estudios de prevalencia de demencia realizados en comunidad, donde los estimados a nivel mundial sugieren que casi dos tercios de los individuos diagnosticados con EA son del sexo femenino ${ }^{(27)}$, de la misma manera en el estudio de prevalencia de demencia realizado en el Cercado de Lima, alcanza el $58,5 \%$ de la muestra estudiada y es mayor $(61,5 \%)$ en el grupo de edades entre 80 y 84 años ${ }^{(4)}$. La edad promedio de los pacientes con demencia también refleja lo que sucede en la comunidad; pues demencia y particularmente EA es dependiente de la edad, así en estudios de LA se ha demostrado que la prevalencia de demencia se incrementa con la edad ${ }^{(3)}$.

En cuanto al rendimiento del MMSE, aunque es la prueba de cribado para demencia la más utilizada en el mundo ${ }^{(7,16)}$, en este análisis de datos de una investigación realizada en comunidad donde los casos y controles fueron establecidos según los resultados de la evaluación neuropsicológica, no ha mostrado resultados satisfactorios: una baja sensibilidad $(64,1 \%)$, y alta proporción de falsos positivos (15,9\%); así mismo, tener una prueba positiva genera un aumento pequeño del $\mathrm{LR}+(4,03)$, mientras que una negativa genera descenso también pequeño del LR-(0,43), esto nos indica que MMSE no es un buen test para el cribado de demencias de cualquier tipo en esta muestra estudiada. Sin embargo, este hecho podría explicarse por la proporción de pacientes con demencia en estadios leves, que fue del $34,9 \%$ y la pobre capacidad del MMSE para captar a pacientes con demencia en etapas tempranas $(7,8,10,33)$, mientras que la estratificación del MMSE por nivel de instrucción también influye en su baja sensibilidad ${ }^{(7)}$. La PDR-M, muestra un mejor rendimiento con respecto al MMSE, pues muestra $81,9 \%$ de sensibilidad, siendo una prueba altamente específica $(98,1 \%)$; así mismo, muestra un gran aumento del LR+ $(47,79)$ cuando la prueba es

Tabla 4. Rendimiento de pruebas cognitivas breves aplicadas en pacientes con demencia de tipo no alzhéimer en Cercado de Lima $(n=210)$

\begin{tabular}{|c|c|c|c|c|c|c|}
\hline \multirow{2}{*}{ Indicador } & \multicolumn{2}{|c|}{ MMSE } & \multicolumn{2}{|c|}{ PDR-M } & \multicolumn{2}{|c|}{ MMSE Y PDR-M } \\
\hline & $\%$ & IC $95 \%$ & $\%$ & IC $95 \%$ & $\%$ & IC $95 \%$ \\
\hline Sensibilidad & 80,0 & $54,8-93,0$ & 86,7 & $62,1-96,3$ & 93,30 & $70,2-98,8$ \\
\hline Especificidad & 84,1 & $76-89,8$ & 98,1 & $93,4-99,5$ & 84,10 & $76-89,8$ \\
\hline Valor predictivo positivo & 28,8 & $19,6-40,1$ & 78,8 & $48,1-93,7$ & 32,0 & $23,0-42,6$ \\
\hline Valor predictivo negativo & 98,1 & $95,0-99,3$ & 98,9 & $96,2-99,7$ & 99,4 & $95,9-99,9$ \\
\hline Área ROC (exactitud) & 0,82 & $0,71-0,93$ & 0,92 & $0,83-1,00$ & 0,89 & $0,81-0,96$ \\
\hline Odds ratio diagnóstica & 21,2 & $5,4-83,11$ & 341,3 & $44,24-2632,21$ & 74,12 & $9,13-601,61$ \\
\hline $\operatorname{LR}(+)$ & 5,0 & $3,04-8,34$ & 46,4 & $11,58-185,63$ & 5,87 & $3,72-9,27$ \\
\hline $\operatorname{LR}(-)$ & 0,2 & $0,09-0,66$ & 0,1 & $0,04-0,49$ & 0,08 & $0,01-0,53$ \\
\hline
\end{tabular}

MMSE: Mini Mental State Examination. PDR-M: prueba de dibujo del reloj - versión de Manos. LR(+): Likehood ratio positivo. LR(-):Likehood ratio negativo. 
positiva, y un descenso moderado del LR- $(0,11)$ cuando la prueba es negativa, estos resultados nos demuestran que la PDR-M es una excelente prueba para el cribado de pacientes con demencia de cualquier tipo; sin embargo, debemos tener en cuenta que los pacientes con demencia de este estudio, se encontraban, en su mayoría, $(60,2 \%)$ en un estadio moderado a severo, donde es frecuente el compromiso de las funciones visuoespaciales (FVE) y construccionales (FC) ${ }^{(17,34)}$. Se ha demostrado que, conforme progresa la EA, las FVE y FC están deterioradas y se puede evidenciar al dibujar y/o copiar el reloj ${ }^{(34)}$. Por otro lado, múltiples estudios han demostrado que diversas versiones de PDR son superiores a MMSE para detectar demencia en diversos estadios, según CDR; pero puede fallar para detectar demencia en estadios leves ${ }^{(16,35)}$. Al analizar la combinación de ambas pruebas, MMSE y PDR-M, observamos que el tener, al menos, uno positivo, tiene una alta sensibilidad $(98,1 \%)$, mayor a la obtenida individualmente; así mismo, el hecho de tener las dos pruebas negativas, tiene un alto VPN, con un mejor resultado de LR-; explicado por el estadio avanzado de la muestra. La notable mejoría del rendimiento de las pruebas en combinación, se debe a que la PDR-M mide, además, funciones frontales (particularmente de funciones ejecutivas como planificación, programación motora y ejecución) y temporoparietales (comprensión auditiva, memoria visual, reconstrucción de una imagen gráfica y habilidades visuoespaciales), que MMSE no realiza ${ }^{(17,34)}$. De esta manera, podemos afirmar que la administración de ambas pruebas es útil en el cribado de demencias. Al realizar el análisis por separado de demencia tipo alzhéimer y no alzhéimer, los valores de los parámetros no se diferencian sustancialmente de los obtenidos para demencias de cualquier tipo, observándose los mejores resultados para la combinación de ambas pruebas.

Las principales limitaciones del presente estudio están relacionadas con la severidad de demencia de la muestra y la ausencia de un análisis de correlación de las PCB con CDR, pues ambos han demostrado fallar en la detección de demencia en estadios leves ${ }^{(32,35)}$. Sin embargo, debemos tener en cuenta que, en nuestro país, los casos de demencia se detectan en estadios moderados a avanzados ${ }^{(4)}$; por lo que pueden ser útiles como pruebas de cribado en atención primaria. Otra limitación estaría relacionada con el hecho de que las PCB (MMSE, PDR-M y PFAQ) fueron empleadas como cribado en la primera fase del estudio de comunidad, lo cual restaría la validez interna de las PCB; sin embargo, la confirmación del deterioro cognitivo fue realizada por un segundo evaluador, quien no realizó la primera evaluación, y la confirmación y tipificación de demencia fue realizada por la evaluación conjunta de neurólogos y neuropsicólogos, lo cual nos permitió seleccionar el grupo de casos y controles a partir del cual se realizó en ciego el rendimiento de las PCB. Por otro lado, en nuestro país se ha validado el T@M que reúne el perfil ideal capaz de detectar deterioro cognitivo leve y EA en estadios iniciales, el cual podría ser utilizado en casos de quejas subjetivas de memoria, sin compromiso de las actividades de vida diaria evidente para familiares y cuidadores.

En conclusión, ambas PCB utilizadas en conjunto, demuestran buena capacidad discriminativa para detectar demencia en estadios moderado y severo en población viviendo en comunidad urbana de Lima. Debido a su buen rendimiento y bajo costo, pueden ser recomendados para realizar en atención primaria.

Contribución de los autores: NC, CA-D, YB-B, RM, DL y SC-S han participado en la concepción del artículo, la recolección de datos, su redacción y aprobación de la versión final. Además, CA-D, YB-B y EH-P realizaron el análisis de datos. JC-A y EV-L participaron como neuropsicólogos ciegos a las evaluaciones de campo.

\section{Conflictos de intereses: ninguno.}

Fuentes de financiamiento: autofinanciado

\section{REFERENCIAS BIBLIOGRÁFICAS}

1. Llibre Rodriguez JJ, Ferri CP, Acosta D, Guerra M, Huang Y, Jacob KS et al. Prevalence of dementia in Latin American, India and China: a population-based cross-sectional survey. Lancet. 2008;372(9637):464474 .

2. Kalaria RN, Maestre GE, Arizaga $R$, Friedland RP, Galasko D, Hall K, et al. Alzheimer's disease and vascular dementia in developing countries: prevalence, management and risk factors. Lancet Neurol. 2008;7(9):812-826.
3. Nitrini R, Bottino C, Albala, C, Custodio N, Ketzoian C, Llibre Rodriguez JJ, et al. Prevalence of dementia in Latin American: a collaborative study of populationbased cohorts. International Psychogeriatrics 2009;21:622-630.

4. Custodio N, García A, Montesinos R, Escobar J \& Bendezú L. Prevalencia de demencia en una población urbana de Lima-Perú: Un estudio puerta a puerta. An Fac med. 2008; 69(4): 233-238.
5. Dubois B, Feldman HH, Jacova C, Dekosky ST, Barberger-Gateau P, Cummings J, et al. Research criteria for the diagnosis of Alzheimer's disease: revising the NINCDS-ADRDA criteria. Lancet Neurol. 2007;6:734-746.

6. Moorhouse P, Rockwood K. Vascular cognitive impairment: current concepts and clinical developments. Lancet Neurol.2008;7:246-255. [Citado el 28 de Febrero de 2016].

7. Villarejo A, Puertas-Martín V. Utilidad de los test breves en el cribado de 
demencia. Neurología. 2011;26:425433.

8. Böhm P, Peña-Casanova J, Gramunt N, Manero RM, Terrón C, QuiñonesÚbeda S. Versión española del Memory Impairment Screen (MIS): datos normativos y de validez discriminativa. Neurología. 2005;20:402—411.

9. Cacho J, García-García R, Arcaya J, Vicente JL, Lantada N. Una propuesta de aplicación y puntuación del test del reloj en la enfermedad de Alzheimer. Rev Neurol. 1999;28:648-655.

10. Borson S, Scanlan J, Brush M, Vitaliano P, Dokmak A. The minicog: a cognitive 'vital signs' measure for dementia screening in multilingual elderly. Int J Geriatr Psychiatry. 2000;15:1021-1027.

11. Solomon PR, Hirschoff A, Kelly B, Relin M, Brush M, DeVeauxRD, et al. A 7 minute neurocognitive screening battery highly sensitive to Alzheimer's disease. Arch Neurol. 1998;55:349_355.

12. Carnero-Pardo C, MontoroRíos MT. Test de las fotos. Rev Neurol.2004;39:801-806.

13. Carnero Pardo C, Sáez-Zea C, Montiel Navarro L, Del Sazo P,Feria Vilar I, Pérez Navarro MJ, et al. Utilidad diagnóstica del test de las fotos (Fototest) en el deterioro cognitivo y demencia. Neurología. 2007;22:860869.

14. Carnero-Pardo C, Gurpegui M, Sánchez-Cantalejo E, Frank A,Mola $\mathrm{S}$, Barquero MS, et al. Diagnostic accuracy of the Eurotest for dementia: a naturalistic, multicenter phase II study. BMCNeurol. 2006;6:15.

15. Rami L, Molinuevo JL, Sanchez-Valle R, Bosch B, Villar A. Screening for amnestic mild cognitive impairment and early Alzheimer's disease with M@T (Memory Alteration Test) in the primary care population. Int $\mathrm{J}$ Geriatr Psychiatry.2007;22:294-304.

16. Custodio N, Lira D. Adaptación Peruana del Mini Mental State Examination (MMSE). An Fac med. 2014; 75(1): 69 .

17. Custodio N, García A, Montesinos R, Lira D \& Bendezú L. Validación de la prueba de dibujo del reloj-versión de Manos como prueba de cribado para detector demencia en una población adulta mayor de Lima, Perú. Rev Peru Med Exp Salud Publica 2011;28(1):29-34.
18. Custodio N, Lira D, Montesinos R, Gleichgerrcht \& Manes F. Utilidad del Addenbrookes's Cognitive Examination versión en español en pacientes peruanos con enfermedad de Alzheimer y demencia frontotemporal. Vertex Rev Arg de Psiquiat 2012; XXIII:165-172.

19. Custodio N, Lira D, Herrera-Perez E, Nuñez del Prado L, Parodi J, GuevaraSilva E, et al. The Memory Alteration Test discriminates between Cognitively Healthy Status, Mild Cognitive Impairment and Alzheimer's Disease. Dementia Geriatric and Cognitive Disorder Extra 2014;4:314-321.

20. Butman J, Arizaga R, Harris P, Drake M, Baumann Denise, de Pascale A, Allegri R, et al. El "Mini -Mental State Examination” en español. Normas para Buenos Aires. Rev Neurol Arg.2001;26:11-5.

21. Quiroga P, Albala C and Klaasen G. Validación de un test de tamizaje para el diagnóstico de demencia asociado a edad, en Chile. Rev Med Chile 2004;132:467-478.

22. American Psychiatric Association. Diagnostic and statistical manual of mental disorders, DSM-IV-TR. 4th ed. Washington, DC: American Psychiatric Association, 2000.

23. McKhann G, Drachman D, Folstein M, Katzman R, Price D, Stadlan EM. Clinical diagnosis of Alzheimer's disease: report of the NINCDSADRDA work group under the auspices of department of health and human services task force on Alzheimer's disease. Neurology 1984;34:939-944.

24. Roman GC, Tatemichi TK, Erkinjuntti T, Cummings JL, Masdeu JC, Garcia JH. Vascular dementia: diagnostic criteria for research studies. Report of the NINDS-AIREN international workshop. Neurology. 1993;43:250260.

25. Neary D, Snowden JS, Gustafson L, Passant U, Stuss D, Black S, et al. Frontotemporal lobar degeneration: a consensus on clinical diagnostic criteria. Neurology. 1998;51:15461554.

26. McKeith IG, Dickson DW, Lowe J, Emre M, O'Brien, Feldman $\mathrm{H}$, et al. Diagnosis and management of dementia with Lewy bodies: Third report of the DLB consortium. Neurology 2005; 65(12): 1863-1872.
27. Rey A. L'examen physiologique dans le cas d'encephalopathie traumatique [Psychological examination in a case of traumatic encephalopathy]. Arch. Psychol 1941;286-340.

28. Wechsler D (1997) Wechsler Memory Scale, Psychological Corporation.

29. Partington JE, Leiter R .Partington's pathway test. Psychol Serv Cent Bull 1949; 9-20.

30. Kaplan E, Goodglass H, Weintraub S, Goodglass H (1983) Boston naming test, Lea \& Febiger, Philadelphia.

31. Nelson HE. A Modified Card Sorting Test Sensitive to Frontal Lobe Defects. Cortex 1976; 12, 313-324.

32. Custodio N, Becerra-Becerra Y, Alva-Diaz C, Montesinos R, Lira D, Herrera-Pérez E, et al. Validación y precisión de una escala de deterioro global para establecer severidad de demencia en una población de Lima. CesMedicina 2016; in press.

33. Mielke MM, Vemuri P \& Rocca WA. Clinical epidemiology of Alzheimer's disease: assessing sex and gender differences. Clin Epidemiol 2014;6:3748.

34. Delgado Derio C, Guerrero Bonnet $S$, Troncoso Ponce M, Araneda Yañez A, Slachevsky Chochol A \& Behrens Pellegrino MI. Memory, fluency, and orientation (MEFO): A five-minute screening test for cognitive decline. Neurologia 2013;28(7):400-407.

35. Nagaratnam N, Nagaratnam K \& $\mathrm{O}^{\prime}$ mara D. Intersecting pentagon copying and clock drawing test in mild and moderate Alzheimer's disease. J Clin Gerontol Geriatr 2014;5:47-52.

36. Powlishta KK, Von Dras DD, Stanford A, Carr DB, Tsering C, Miller JP, et al. The clock drawing test is a poor screen for very mild dementias. Neurology 2002;59:898-903.

\footnotetext{
Correspondencia: Nilton Custodio.

Unidad de Investigación. Instituto Peruano de Neurociencias.Bartolomé Herrera 161. Lince.

51-1-2653834.

Correo electrónico:ncustodio@ipn.pe
} 